\title{
Differentiating prostate cancer from benign prostatic hyperplasia using whole-lesion histogram and texture analysis of diffusion- and T2-weighted imaging
}

Pengyi Xing ${ }^{1+}$, Luguang Chen ${ }^{1+}$ (D), Qingsong Yang ${ }^{1}$, Tao Song ${ }^{1}$, Chao Ma ${ }^{1}$, Robert Grimm² ${ }^{2}$ Caixia Fu ${ }^{3}$, Tiegong Wang ${ }^{1}$, Wenjia Peng ${ }^{1}$ and Jianping $L^{1{ }^{*}}$

\begin{abstract}
Background: To explore the usefulness of analyzing histograms and textures of apparent diffusion coefficient (ADC) maps and T2-weighted (T2W) images to differentiate prostatic cancer (PCa) from benign prostatic hyperplasia $(\mathrm{BPH})$ using histopathology as the reference.

Methods: Ninety patients with PCa and 112 patients with BPH were included in this retrospective study. Differences in whole-lesion histograms and texture parameters of ADC maps and T2W images between PCa and $\mathrm{BPH}$ patients were evaluated using the independent samples t-test. The diagnostic performance of ADC maps and T2W images in being able to differentiate PCa from BPH was assessed using receiver operating characteristic (ROC) curves.

Results : The mean, median, $5^{\text {th }}$, and $95^{\text {th }}$ percentiles of ADC values in images from PCa patients were significantly lower than those from BPH patients $(p<0.05)$. Significant differences were observed in the means, standard deviations, medians, kurtosis, skewness, and $5^{\text {th }}$ percentile values of T2W image between PCa and BPH patients $(p<$ 0.05). The $A D C_{5 \text { th }}$ showed the largest AUC (0.906) with a sensitivity of $83.3 \%$ and specificity of $89.3 \%$. The diagnostic performance of the T2W image histogram and texture analysis was moderate and had the largest AUC of 0.634 for $T 2 W_{\text {Kurtosis }}$ with a sensitivity and specificity of $48.9 \%$ and $79.5 \%$, respectively. The diagnostic performance of the combined $A D C_{5 \text { th }} \& T 2 W_{\text {Kurtosis }}$ parameters was also similar to that of the $A D C_{5 \text { th }} \& A D C_{\text {Diff }}$ -Variance.

Conclusions: Histogram and texture parameters derived from the ADC maps and T2W images for entire prostatic lesions could be used as imaging biomarkers to differentiate PCa and BPH biologic characteristics, however, histogram parameters outperformed texture parameters in the diagnostic performance.
\end{abstract}

Keywords: Prostate cancer, Prostatic Hyperplasia, Magnetic resonance imaging, Diffusion

\footnotetext{
*Correspondence: cj.lujianping@vip.163.com

${ }^{\dagger}$ Pengyi Xing and Luguang Chen contributed equally.

'Department of Radiology, Changhai Hospital of Shanghai, The Second Military Medical University, No.168 Changhai Road, 200433 Shanghai, China

Full list of author information is available at the end of the article
}

(c) The Author(s). 2021 Open Access This article is licensed under a Creative Commons Attribution 4.0 International License, which permits use, sharing, adaptation, distribution and reproduction in any medium or format, as long as you give appropriate credit to the original author(s) and the source, provide a link to the Creative Commons licence, and indicate if changes were made. The images or other third party material in this article are included in the article's Creative Commons. licence, unless indicated otherwise in a credit line to the material. If material is not included in the article's Creative Commons licence and your intended use is not permitted by statutory regulation or exceeds the permitted use, you will need to obtain permission directly from the copyright holder. To view a copy of this licence, visit http://creativecommons.org/licenses/by/4.0/ The Creative Commons Public Domain Dedication waiver (http://creativecommons.org/publicdomain/zero/1.0/) applies to the data made available in this article, unless otherwise stated in a credit line to the data. 


\section{Background}

Prostate cancer (PCa) is the second most commonly diagnosed cancer in men worldwide [1]. Patients with suspected PCa usually undergo a standard transrectal ultrasound-guided biopsy. However, increased serum prostate-specific antigen (PSA) levels and abnormal digital rectal examinations have also been used as detection methods. Unfortunately, these tests have resulted in the insufficient detection of high-grade PCa tumors and excessive detection of low-grade lesions [2]. Although a few randomized-controlled trials have shown that patients with low-grade tumors failed to benefit from therapy, most patients continued to have excessive therapeutic interventions and follow-up examinations, which increased patient and the healthcare sector medical costs $[3,4]$.

With the increasing application of multi-parametric magnetic resonance imaging (mpMRI), more focal PCa tumors can be detected and accurately localized, making early and precise PCa therapies possible [5]. Diffusionweighted imaging (DWI) is a noninvasive technique used to evaluate the microscopic mobility of water molecules in tissues and has been used to detect and evaluate prostatic tumors [6, 7]. Apparent diffusion coefficient (ADC) maps, derived from DWI images, can reflect the histologic characteristics of lesions and has enhanced $\mathrm{PCa}$ diagnosis as a supplementary diagnostic tool [8]. Kuhl et al. found that bi-parametric MRI (bpMRI, T2weighted imaging, and DWI) and mpMRI (T2WI, DWI, and dynamic contrast-enhanced MRI) had similar diagnostic efficiency and accuracy. bPMRI image interpretations were also have a good consistency among radiologists, and the diagnostic accuracy of tumor detection was similar to that of mpMRI. However, bpMRI had significantly shorter imaging acquisition and interpretation time, and no contrast agent is needed compared with mpMRI [9]. Dynamic contrast-enhanced (DCE) MRI characterizes the pharmacokinetic tissue properties through imaging during the administration of contrast agent. However, this method has several limitations, such as potential adverse reactions to gadolinium administration, additional scanning time and cost, and poor consistency in the interpretation of images among radiologists, limiting its broad application in clinical practice $[10,11]$.

Image texture analysis can be used to estimate the heterogeneity of image signals by quantifying the roughness and regularity of grayscale pixel value spatial distributions in normal and pathologic tissues; the macroscopic heterogeneity of images might reflect microscopic heterogeneity at the level of histopathology $[12,13]$. Several studies have shown that MR image texture analysis could detect, classify, evaluate, and predict breast, brain, rectal, and cervical cancer lesions [14-17]. Sidhu et al. used single-slice texture analysis of ADC, T2W, and contrast-enhanced T1W images to identify clinically significant carcinomas in patients with transitional prostatic lesions [18]. Wibmer et al. found that Haralick texture analysis of prostate MRI could be used to detect PCa and differentiate Gleason scores [19]. Differentiating $\mathrm{PCa}$ from benign prostatic hyperplasia $(\mathrm{BPH})$ remains a challenge using conventional multi-parametric MRI due to lesion heterogeneity. Histogram and texture image analysis is a promising tool that provides a numerical representation of data distributions and is particularly useful when evaluating the heterogeneous features of tumors [14, 20, 21]. Cui et al. evaluated the diagnostic performance of histogram analysis of intravoxel incoherent motion parameters for differentiating $\mathrm{PCa}$ from $\mathrm{BPH}$ [22]. Chatterjee et al. found that ADC values were significantly lower in $\mathrm{PCa}$ compared to all $\mathrm{BPH}$ types and can differentiate between $\mathrm{PCa}$ and $\mathrm{BPH}$ with high accuracy (areas under the curve: $\mathrm{AUC}=0.87$ ) [23]. Bonekamp et al. compared biparametric contrast-free radiomic machine learning, mean $\mathrm{ADC}$, and radiologist assessment for characterization of prostate lesions detected during prospective MRI interpretation. They reported an AUC of 0.84 for mean ADC values and validated their results with a test cohort of 133 patients [24]. Peng et al. reported AUC values for the differentiation of $\mathrm{PCa}$ from normal foci of the $10^{\text {th }}$ percentile ADC, average ADC, T2-weighted skewness, and Ktrans [25]. However, using whole-lesion histogram and texture analysis with bpMRI to distinguish $\mathrm{BPH}$ nodules from $\mathrm{PCa}$ has not yet been reported.

Therefore, the study aimed to explore the usefulness of ADC map and T2W image histogram and texture analyses to distinguish $\mathrm{PCa}$ from $\mathrm{BPH}$ using histopathology as the reference.

\section{Methods \\ Subjects}

This retrospective study was approved by the local institutional review board (NO. M20140149), and individual consent for this retrospective analysis was waived. Between March 2015 and July 2017, consecutive patients with pathologically proven $\mathrm{PCa}$ or $\mathrm{BPH}$ were enrolled in this study. Patients who met the following criteria were included in the present study: (1) had pathologically proven prostatic hyperplasia (systemic biopsy) or cancer (prostatectomy); (2) had prostatic MRI examinations performed; (3) the interval between prostatic biopsy/radical resection and MRI was less than 3 months; and (4) no history of other malignant tumors. The exclusion criteria were as follows: (1) histopathology of lesion biopsies were confirmed to be positive but were negative on MRI; (2) pretreatments were given to treat prostatic lesions, such as endocrine, chemotherapy, or radiotherapy; 
(3) poor image quality due to motion artifacts or severe susceptibility artifacts; and (4) incomplete imaging protocol, images of DWI or T2W were missed to perform histogram and texture analysis. Patient clinical characteristics were recorded, including age, PSA levels, lesion volumes, score of Prostate Imaging Reporting and Data System (PI-RADS, version 2), and Gleason scores (in the case of lesions was confirmed to be $\mathrm{PCa}$ ).

\section{Magnetic resonance imaging}

All imaging was performed on a 3 Tesla MRI system (MAGNETOM Skyra, Siemens Healthcare, Erlangen, Germany) using a standard 18-channel phased-array body coil and 32-channel integrated spine coil. The main parameters of axial DWI were repetition time/echo time $(\mathrm{TR} / \mathrm{TE})=5100 / 89 \mathrm{~ms}$, field of view $(\mathrm{FOV})=224 \times 280$ $\mathrm{mm}^{2}$, matrix $=120 \times 150$, slices $=20$, slice thickness $=4$ $\mathrm{mm}$, gap $=0 \mathrm{~mm}$, acceleration factor $=2, b$-values (number of averages) $=0$ (1), 500(2), 800(4), 1000(5), 1500(6), $2000(8) \mathrm{s} / \mathrm{mm}^{2}$, diffusion gradients applied in three orthogonal directions, and acquisition times $=6 \mathrm{~min} 43 \mathrm{~s}$. ADC maps were inline calculated using the monoexponential model, $S(b)=S(0) e^{-b^{*} A D C}$, where $S(b)$ is the signal intensity with a $b$-value $>0$, and $S(0)$ is the signal intensity with a $b$-value $=0$. Parameters for axial T2weighted turbo spin echo sequence were $\mathrm{TR} / \mathrm{TE}=5460$ / $104 \mathrm{~ms}, \quad$ FOV $=180 \times 180 \mathrm{~mm}^{2}, \quad$ matrix $=384 \times 384$, slices $=24$, slice thickness $=4 \mathrm{~mm}$, gap $=0 \mathrm{~mm}$, echo train length $=18$, and acquisition time $=3 \mathrm{~min} 49 \mathrm{~s}$.

\section{Image analysis}

All images were sent to a dedicated workstation for data processing and were independently assessed by two experienced radiologists (P.X. and Q.Y.) with 6 and 8 years of experience in pelvic radiology, respectively. The radiologists were blinded to the data and clinical information, and using a consensus, selected the largest lesions in patients with multicentric or multifocal tumors for further analysis.

Whole-lesion histogram and texture analyses were performed on ADC maps and T2W images with the prototypic MR Multiparametric Analysis software (Siemens Healthcare, Erlangen, Germany) by the radiologists using the following steps: (1) Import data. T2W images, DWI images with $\mathrm{b}=1500 \mathrm{~s} / \mathrm{mm}^{2}$, and ADC maps were loaded into the histogram and texture analysis software. (2) Region of interest (ROI) delineations were acquired. Foreground and background seed points were manually drawn inside and outside of lesions, respectively, on three reformatted planes of the DWI images. Then, they were automatically copied to ADC maps and T2W images. (3) Lesion segmentation. Segmentation of the whole lesion was performed based on seed points with a random walker algorithm [14]. Manual adjustments for segmentation were performed, if necessary. (4) Histogram and texture analysis. Histogram and texture analyses for entire lesions on the ADC maps and T2W images were performed, and statistical parameters were extracted, including lesion volume, mean, standard deviation, median, $5^{\text {th }}$ and $95^{\text {th }}$ percentiles, differential variance (diff-variance), differential entropy (diff-entropy), contrast, entropy, skewness, and kurtosis. Figure 1 shows the workflow of histogram and texture analysis.

\section{Histopathologic analysis}

All patients underwent transrectal ultrasound-guided biopsy or radical prostatectomy. Biopsies and prostatectomies were formalin-fixed and subjected to tissue sectioning. Tissue sections were stained with a hematoxylin \& eosin stain and subjected to immunohistochemical analyses. A urologic pathologist with 8 years of experience, observed the histologic sections and determined lesion locations and boundaries. If the lesions were confirmed to be PCa, Gleason scores were determined according to the $\mathrm{PCa}$ grading guidelines formulated by the 2014 Consensus of the International Urological Pathology Association [26]. For patients with $\mathrm{BPH}$, the largest hyperplastic nodules were selected for analysis, while those with PCa had the largest lesions chosen for analysis.

\section{Statistical analysis}

Statistical analyses were performed using SPSS software (Version 19, IBM Statistical Package for the Social Sciences, Chicago, IL) and MedCalc (Version 18.2.1, MedCalc Software, Mariakerke, Belgium). Quantitative variables are presented as the mean \pm standard deviation or as the median ( $1^{\text {st }}$ and $3^{\text {rd }}$ quartile ranges) based on the normal distribution of the data, and the categorical variables are expressed as percentages. Differences in PIRADS score, PSA levels, and lesion volumes between the $\mathrm{PCa}$ and $\mathrm{BPH}$ patients were compared using MannWhitney U tests. Statistical variable differences in age, histograms and texture analyses of the ADC maps and T2W images between $\mathrm{PCa}$ and $\mathrm{BPH}$ patients were evaluated using the independent-samples t-test. In addition, the diagnostic performance of histogram and texture parameters on $\mathrm{ADC}$ maps and $\mathrm{T} 2 \mathrm{~W}$ images in being able to differentiate $\mathrm{PCa}$ from $\mathrm{BPH}$ was assessed using receiver operating characteristic (ROC) curves, and the $95 \%$ confidence interval (CI) for the area can be used to test the hypothesis that the theoretical area is 0.5 . If the CI does not include the 0.5 value, then there is evidence that the laboratory test does have an ability to distinguish between the two groups [27, 28]. Furthermore, we explored the diagnostic performance of optimized and combined parameters, based on the best diagnostic 


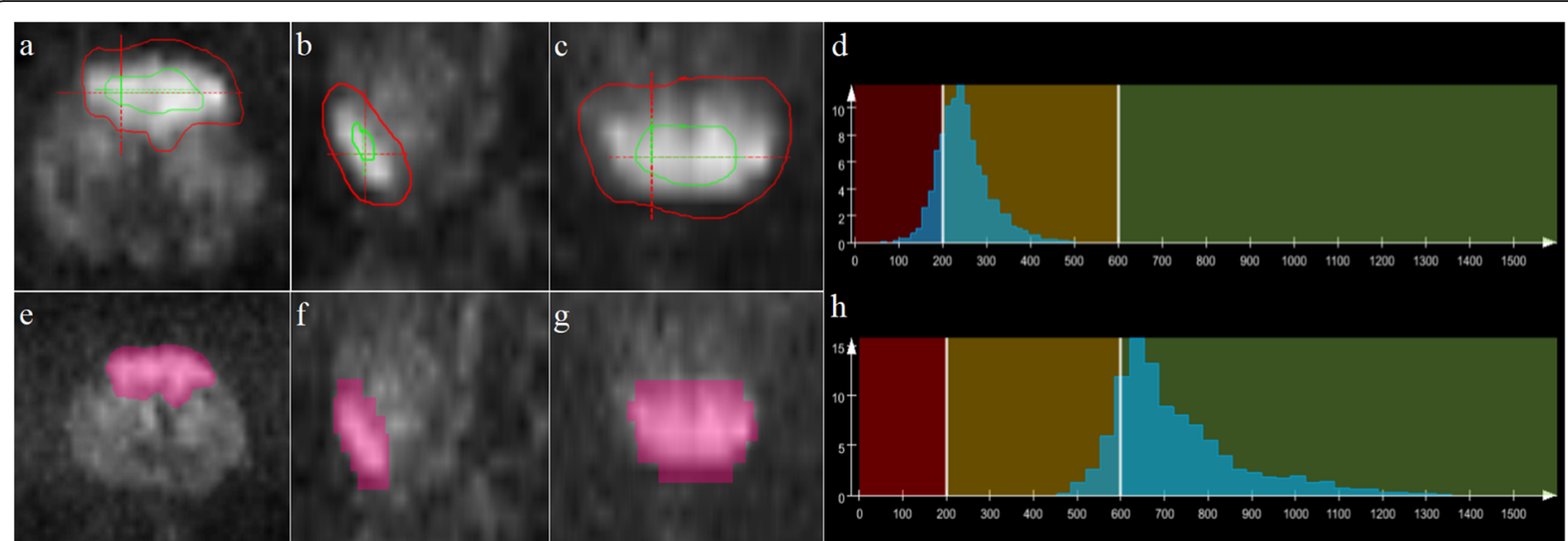

Fig. 1 Flow diagrams of the whole-lesion histogram and texture analysis. a-c: Foreground and background seed points were manually drawn inside (green color) and outside (red color) on three reformatted diffusion-weighted imaging (DWI) images with $b=1500 \mathrm{~s} / \mathrm{mm}^{2}$. e-g: Threedimensional segmentations were generated on the DWI images. $\mathbf{d}$, $\mathbf{h}$ : Histograms of the T2weighted (T2W) images and apparent diffusion coefficient (ADC) maps that were generated

performance of the ADC map and/or T2W image histogram and texture parameters. A $p$-value $<0.05$ was considered statistically significant.

\section{Results}

\section{Patients}

Two hundred and thirteen patients with prostatic lesions were enrolled in this study. Among these, 11 patients were excluded because three had poor image quality, two had lesions confirmed to be positive by histopathologic diagnoses but had negative MRI results, four had endocrine, chemotherapy, or radiotherapy before MRI, and two had incomplete imaging protocol. Finally, a total of 202 patients with a mean age of $65.9 \pm 8.7$ years (range 37-86 years) were included in the final analysis. Ninety $(44.5 \%)$ patients were diagnosed with $\mathrm{PCa}$, and $112(55.5 \%)$ were diagnosed with $\mathrm{BPH}$. There were significant differences between $\mathrm{PCa}$ and $\mathrm{BPH}$ patients in median scores (quartile range) of PI-RADS $(5(4,5)$ vs. 2 $(2,2), p<0.0001)$, age (68.9 \pm 7.4 vs. $63.5 \pm 8.9$ years, $p<$ $0.001)$, PSA concentrations $(14.88(9.05,30.13)$ vs. 9.81 $(7.26,15.01) \mathrm{ng} / \mathrm{ml}, p<0.001)$, and lesion volumes $(2.50$ $(1.10,6.00)$ vs. $\left.1.05(0.70,1.70) \mathrm{cm}^{3}, p<0.001\right)$ (Table 1$)$. The number of PCa patients with Gleason scores of $3+$ $3,3+4,4+3$ and $\geq 8$ were $27(13.4 \%), 17(8.4 \%), 14$ $(6.9 \%)$ and $32(15.8 \%)$, respectively.

\section{Comparisons of histogram and texture parameters}

The statistical results regarding differences in histogram and texture parameters of ADC maps and T2W images in patients with $\mathrm{PCa}$ and $\mathrm{BPH}$ are summarized in Table 2. Histogram parameters of the mean, median, and $5^{\text {th }}$ and $95^{\text {th }}$ percentiles of ADC maps were significantly lower in PCa patients compared with those in
BPH patients (all $p<0.0001$ ). For ADC maps, aside from kurtosis $(0.419 \pm 1.212$ vs. $0.315 \pm 1.311, p=0.386)$, standard deviation, diff-variance, diff-entropy, contrast, entropy, and skewness measures were significantly larger in PCa patients than in BPH patients (all $p<0.05)$. Significant differences in the means, standard deviations, medians, kurtosis and skewness values, and $5^{\text {th }}$ percentiles of T2W images were found between $\mathrm{PCa}$ and $\mathrm{BPH}$ patients (all $p<0.05$ ), while no significant differences were observed in the $5^{\text {th }}$ percentile, diff-variance, diffentropy, entropy or contrast parameters.

Table 1 Patient characteristics

\begin{tabular}{|c|c|c|}
\hline \multirow[t]{2}{*}{ Variable } & \multicolumn{2}{|l|}{ Value } \\
\hline & $\mathrm{PCa}$ & $\mathrm{BPH}$ \\
\hline Patients $(n, \%)$ & $90(44.5)$ & $112(55.5)$ \\
\hline Age (years) ${ }^{a}$ & $68.9 \pm 7.4$ & $63.5 \pm 8.9$ \\
\hline PSA level (ng/ml) ${ }^{b}$ & $14.88(9.05,30.13)$ & $9.81(7.26,15.01)$ \\
\hline Lesion volume $(\mathrm{ml})^{\mathrm{b}}$ & $2.50(1.10,6.00)$ & $1.05(0.70,1.70)$ \\
\hline PI-RADS score ${ }^{b}$ & $5(4,5)$ & $2(2,2)$ \\
\hline \multicolumn{3}{|l|}{ Gleason score } \\
\hline $3+3(n, \%)$ & $27(13.4)$ & / \\
\hline $3+4(n, \%)$ & $17(8.4)$ & / \\
\hline $4+3(n, \%)$ & $14(6.9)$ & / \\
\hline$\geq 8(n, \%)$ & $32(15.8)$ & / \\
\hline
\end{tabular}

$P C a$ prostate cancer, $B P H$ benign prostatic hypertrophy, $P S A$ prostate-specific antigen, PI-RADS prostate imaging reporting and data system

${ }^{\mathrm{a}}$, and ${ }^{\mathrm{b}}$, data are expressed as the mean \pm standard deviation and the median $\left(1^{\text {st }}\right.$ and $3^{\text {rd }}$ quartile ranges), respectively 
Table 2 Histogram parameters of apparent diffusion coefficient (ADC) maps and T2weighted (T2W) images in patients with prostate cancer (PCa) and benign prostatic hyperplasia (BPH)

\begin{tabular}{|c|c|c|c|c|}
\hline & Parameters & PCa $(n=90)$ & $\mathrm{BPH}(n=112)$ & $p$-value \\
\hline \multirow[t]{11}{*}{$A D C$} & $5^{\text {th }}$ percentile & $557.661 \pm 131.688$ & $795.973 \pm 116.08$ & $<0.0001$ \\
\hline & Mean & $806.754 \pm 131.268$ & $988.752 \pm 106.763$ & $<0.0001$ \\
\hline & Median & $788.539 \pm 140.309$ & $979.942 \pm 111.27$ & $<0.0001$ \\
\hline & Std & $176.311 \pm 48.874$ & $129.004 \pm 45.47$ & $<0.0001$ \\
\hline & Diff-Variance & $0.195 \pm 0.032$ & $0.178 \pm 0.04$ & $<0.0001$ \\
\hline & Diff-Entropy & $0.757 \pm 0.099$ & $0.707 \pm 0.086$ & $<0.0001$ \\
\hline & Contrast & $0.628 \pm 0.231$ & $0.488 \pm 0.21$ & $<0.0001$ \\
\hline & Entropy & $1.748 \pm 0.289$ & $1.605 \pm 0.234$ & $<0.0001$ \\
\hline & $95^{\text {th }}$ percentile & $1123.756 \pm 169.511$ & $1217.362 \pm 142.675$ & $<0.0001$ \\
\hline & Skewness & $0.472 \pm 0.597$ & $0.297 \pm 0.575$ & 0.026 \\
\hline & Kurtosis & $0.419 \pm 1.212$ & $0.315 \pm 1.311$ & 0.386 \\
\hline \multirow[t]{11}{*}{$\mathrm{T} 2 \mathrm{~W}$} & Kurtosis & $2.272 \pm 2.179$ & $1.375 \pm 1.598$ & 0.001 \\
\hline & Skewness & $0.851 \pm 0.558$ & $0.631 \pm 0.413$ & 0.001 \\
\hline & $5^{\text {th }}$ percentile & $171.594 \pm 43.653$ & $193.165 \pm 45.757$ & 0.001 \\
\hline & Median & $259.822 \pm 54.330$ & $278.165 \pm 54.817$ & 0.014 \\
\hline & Std & $68.67 \pm 20.296$ & $61.937 \pm 18.164$ & 0.012 \\
\hline & Mean & $267.630 \pm 56.500$ & $283.756 \pm 55.486$ & 0.039 \\
\hline & Entropy & $2.355 \pm 0.291$ & $2.362 \pm 0.279$ & 0.797 \\
\hline & Diff-Variance & $0.589 \pm 0.178$ & $0.570 \pm 0.162$ & 0.578 \\
\hline & $95^{\text {th }}$ percentile & $391.011 \pm 88.876$ & $393.134 \pm 78.278$ & 0.759 \\
\hline & Diff-Entropy & $1.467 \pm 0.158$ & $1.459 \pm 0.157$ & 0.808 \\
\hline & Contrast & $1.847 \pm 0.689$ & $1.851 \pm 0.665$ & 0.954 \\
\hline
\end{tabular}

Std standard deviation, Diff-Variance difference in variance, Diff-Entropy difference in entropy

The diagnostic performance of the histogram and texture parameters

Table 3 shows that the ROC results of ADC map qualitative histogram and texture analyses were able to differentiate PCa from BPH. The AUCs for the ADC maps ranged from 0.536 to 0.906 with a sensitivity and specificity of $53.3-83.3 \%$ and $57.1-89.3 \%$, respectively. Moreover, the $5^{\text {th }}$ percentile of the ADC maps showed the largest AUC (0.906) with a sensitivity and specificity of 83.3 and $89.3 \%$, respectively, while kurtosis had the lowest AUC (0.536) with a sensitivity and specificity of 53.3 and $57.1 \%$, respectively. Histogram and texture analysis workflow are shown with two representative $\mathrm{PCa}$ and $\mathrm{BPH}$ patients (Figs. 2 and 3). ROC curve analyses for the histogram and texture parameters of ADC maps and T2W images are shown in Fig. 4. Compared with ADC maps, the diagnostic performance of T2W images in the histogram and texture analyses was moderate, while kurtosis had the largest AUC of 0.634 with a sensitivity and specificity of $48.89 \%$ and $79.46 \%$, respectively (Table 3; Fig. 5). In addition, the diagnostic performance of combining the $5^{\text {th }}$ percentile of the $\mathrm{ADC}$ values $\left(\mathrm{ADC}_{5 \mathrm{th}}\right)$ \& the kurtosis of T2W (T2 $\mathrm{W}_{\text {Kurtosis }}$ ) parameters was the same as that of the combined $\mathrm{ADC}_{5 \text { th }} \& \mathrm{ADC}_{\text {Diff-Variance }}$ parameters, yielding AUCs of 0.906 (95\% CI 0.857, 0.943 ), and sensitivities and specificities of $83.3 \%$, and $89.3 \%$, respectively. However, these combined parameters were not better than the $\mathrm{ADC}_{5 \text { th }}$ parameter alone in distinguishing $\mathrm{PCa}$ from $\mathrm{BPH}$, which was also true of the $\mathrm{T}_{2} \mathrm{~W}_{5 \text { th }} \& \mathrm{~T} 2 \mathrm{~W}_{\text {Diff - Variance combined parameters }}$ (Table 4; Fig. 6).

\section{Discussion}

In the present study, we evaluated the whole-lesion histogram and texture analyses of ADC maps and T2W images to distinguish $\mathrm{PCa}$ and $\mathrm{BPH}$ using histopathologic diagnoses as the reference standard and found those parameters could serve as useful biologic characterizations of $\mathrm{PCa}$. The results demonstrated that histogram and texture analyses of parameters from ADC and T2W images could be useful to differentiate PCa from $\mathrm{BPH}$, however, histogram parameters outperformed texture parameters in the diagnostic performance. All histogram and texture parameters, except for kurtosis, were significantly different in ADC values between $\mathrm{PCa}$ and $\mathrm{BPH}$ patients. Significant differences were observed in 
Table 3 Receiver operating characteristic curve results regarding the qualitative analysis of apparent diffusion coefficient (ADC) maps and T2weighted (T2W) images to distinguish prostate cancer from benign prostatic hyperplasia

\begin{tabular}{|c|c|c|c|c|c|c|c|c|c|}
\hline & Parameters & AUC $(95 \% \mathrm{Cl})$ & Sensitivity (\%) & Specificity (\%) & Cutoff value & $p$ value & Youden index & $+\mathrm{LR}$ & -LR \\
\hline \multirow[t]{11}{*}{$A D C$} & $5^{\text {th }}$ percentile & $0.906(0.858,0.943)$ & 83.3 & 89.3 & $\leq 650.5$ & $<0.0001$ & 0.7262 & 7.78 & 0.19 \\
\hline & Mean & $0.866(0.811,0.910)$ & 73.3 & 92.0 & $\leq 858.379$ & $<0.0001$ & 0.653 & 9.13 & 0.29 \\
\hline & Median & $0.861(0.805,0.905)$ & 68.9 & 93.7 & $\leq 830.5$ & $<0.0001$ & 0.6264 & 11.02 & 0.33 \\
\hline & Std & $0.797(0.734,0.850)$ & 74.4 & 75.0 & $>142.175$ & $<0.0001$ & 0.4944 & 2.98 & 0.34 \\
\hline & Diff-Variance & $0.717(0.649,0.778)$ & 78.9 & 61.6 & $>0.18$ & $<0.0001$ & 0.405 & 2.05 & 0.34 \\
\hline & Diff-Entropy & $0.717(0.650,0.778)$ & 63.3 & 78.6 & $>0.746$ & $<0.0001$ & 0.419 & 2.96 & 0.47 \\
\hline & Contrast & $0.712(0.644,0.773)$ & 67.8 & 69.6 & $>0.506$ & $<0.0001$ & 0.3742 & 2.23 & 0.46 \\
\hline & Entropy & $0.681(0.612,0.744)$ & 70.0 & 64.3 & $>1.668$ & $<0.0001$ & 0.3429 & 1.96 & 0.47 \\
\hline & $95^{\text {th }}$ percentile & $0.674(0.605,0.738)$ & 57.8 & 73.2 & $\leq 1135.5$ & $<0.0001$ & 0.3099 & 2.16 & 0.58 \\
\hline & Skewness & $0.591(0.520,0.660)$ & 65.6 & 54.5 & $>0.299$ & 0.0250 & 0.2002 & 1.44 & 0.63 \\
\hline & Kurtosis & $0.536(0.464,0.606)$ & 53.3 & 57.1 & $>0.089$ & 0.3910 & 0.1048 & 1.24 & 0.82 \\
\hline \multirow[t]{11}{*}{$\mathrm{T} 2 \mathrm{~W}$} & Kurtosis & $0.634(0.563,0.700)$ & 48.89 & 79.46 & $>2.082$ & 0.0008 & 0.2835 & 2.38 & 0.64 \\
\hline & Skewness & $0.633(0.562,0.699)$ & 60 & 66.96 & $>0.653$ & 0.0009 & 0.2696 & 1.82 & 0.6 \\
\hline & $5^{\text {th }}$ percentile & $0.633(0.562,0.699)$ & 82.22 & 41.07 & $\leq 206.5$ & 0.0007 & 0.2329 & 1.4 & 0.43 \\
\hline & Median & $0.601(0.530,0.669)$ & 70 & 52.68 & $\leq 278.5$ & 0.012 & 0.2268 & 1.48 & 0.57 \\
\hline & Std & $0.603(0.532,0.671)$ & 53.33 & 68.75 & $>66.431$ & 0.0119 & 0.2208 & 1.71 & 0.6 \\
\hline & Mean & $0.584(0.513,0.653)$ & 71.11 & 49.11 & $\leq 288.803$ & 0.0371 & 0.2022 & 1.4 & 0.59 \\
\hline & Entropy & $0.511(0.439,0.581)$ & 43.33 & 69.64 & $\leq 2.29$ & 0.7993 & 0.1298 & 1.43 & 0.8 \\
\hline & Diff-Variance & $0.523(0.452,0.593)$ & 25.56 & 82.14 & $>0.711$ & 0.5799 & 0.07698 & 1.43 & 0.9 \\
\hline & $95^{\text {th }}$ percentile & $0.513(0.441,0.583)$ & 88.89 & 3.57 & $\leq 515.5$ & 0.7625 & 0.0754 & 0.92 & 3. \\
\hline & Diff-Entropy & $0.510(0.439,0.581)$ & 42.22 & 65.18 & $>1.526$ & 0.8086 & 0.07401 & 1.21 & 0.8 \\
\hline & Contrast & $0.502(0.431,0.573)$ & 32.22 & 74.11 & $\leq 1.426$ & 0.954 & 0.0633 & 1.24 & 0.5 \\
\hline
\end{tabular}

AUC area under the curve, Std standard deviation, Diff-Variance difference in variance, Diff-Entropy difference in entropy

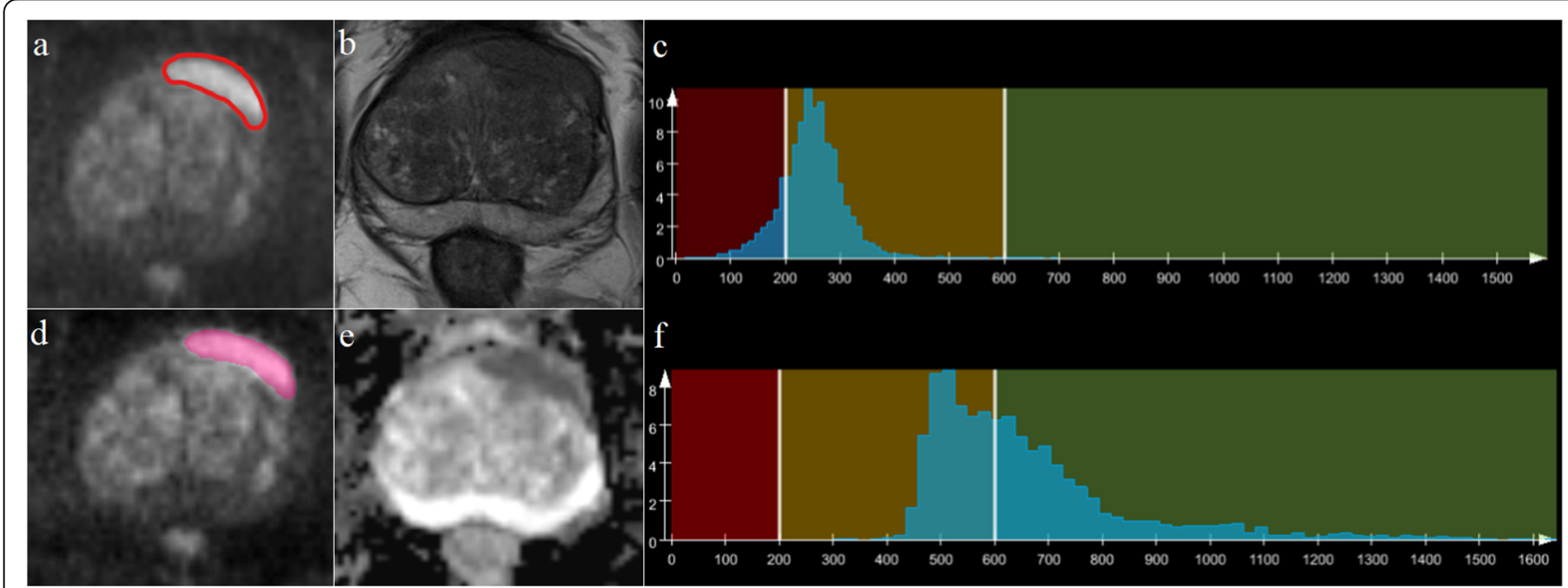

Fig. 2 Representative images of a 69-year-old male with prostatic carcinoma (a-f) and Gleason scores of $4+3$. Segmentation of the lesion is shown on diffusion-weighted imaging (DWI) images with a $b=1500 \mathrm{~s} / \mathrm{mm}^{2}(\mathbf{a}$, d). Histograms (c, f) of T2weighted (T2W) images (b) and ADC maps (e) 


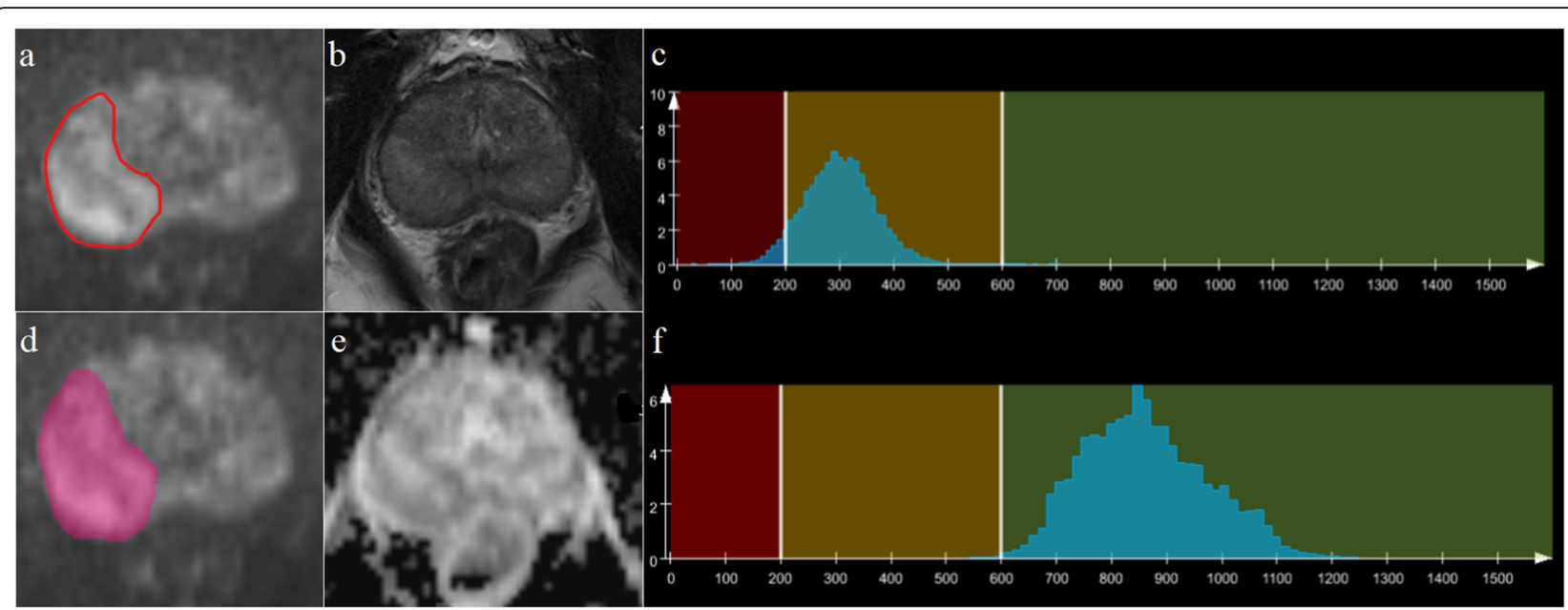

Fig. 3 Representative images of a 63-year-old-male patient with benign prostatic hypertrophy (a-f). Segmentation of the lesion is shown on diffusion-weighted imaging (DWI) images with a $b=1500 \mathrm{~s} / \mathrm{mm}^{2}(\mathbf{a}$, d). Histograms (c, f) of T2weighted (T2W) images (b) and apparent diffusion coefficient (ADC) maps (e) were inline-generated

the means, standard deviations, medians, kurtosis and skewness values, and the $5^{\text {th }}$ percentile of $\mathrm{T} 2 \mathrm{~W}$ images between PCa and BPH lesions.

DWI detects the Brownian motion of water molecules and closely reflects tumor microenvironments, such as tumor cell densities, water content, the amount of fibrous stroma, and cell membrane integrities [29]. ADC maps can provide in vivo quantitative diffusion measurements. Several studies have shown that ADC values were negatively correlated with Gleason classifications [30-

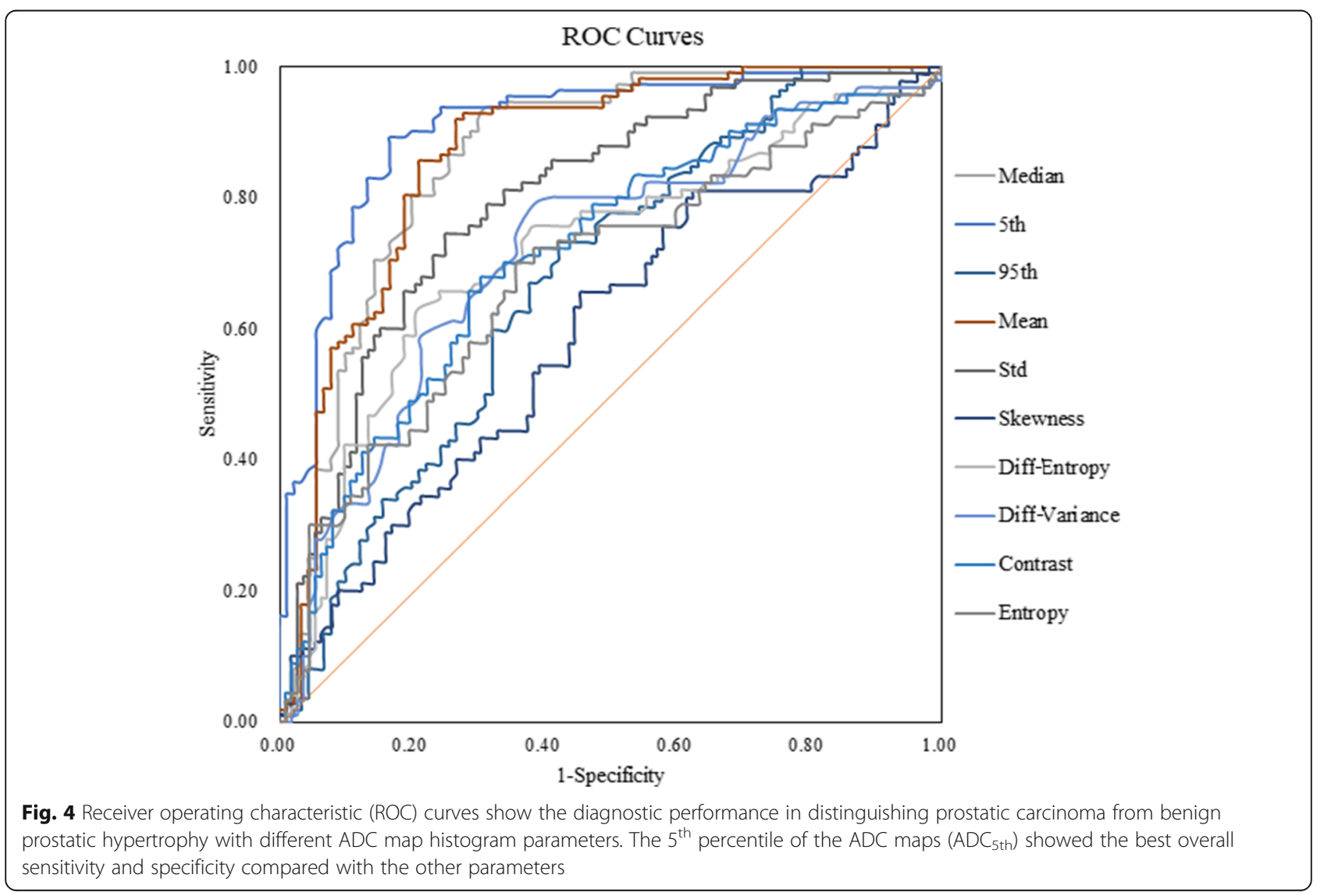




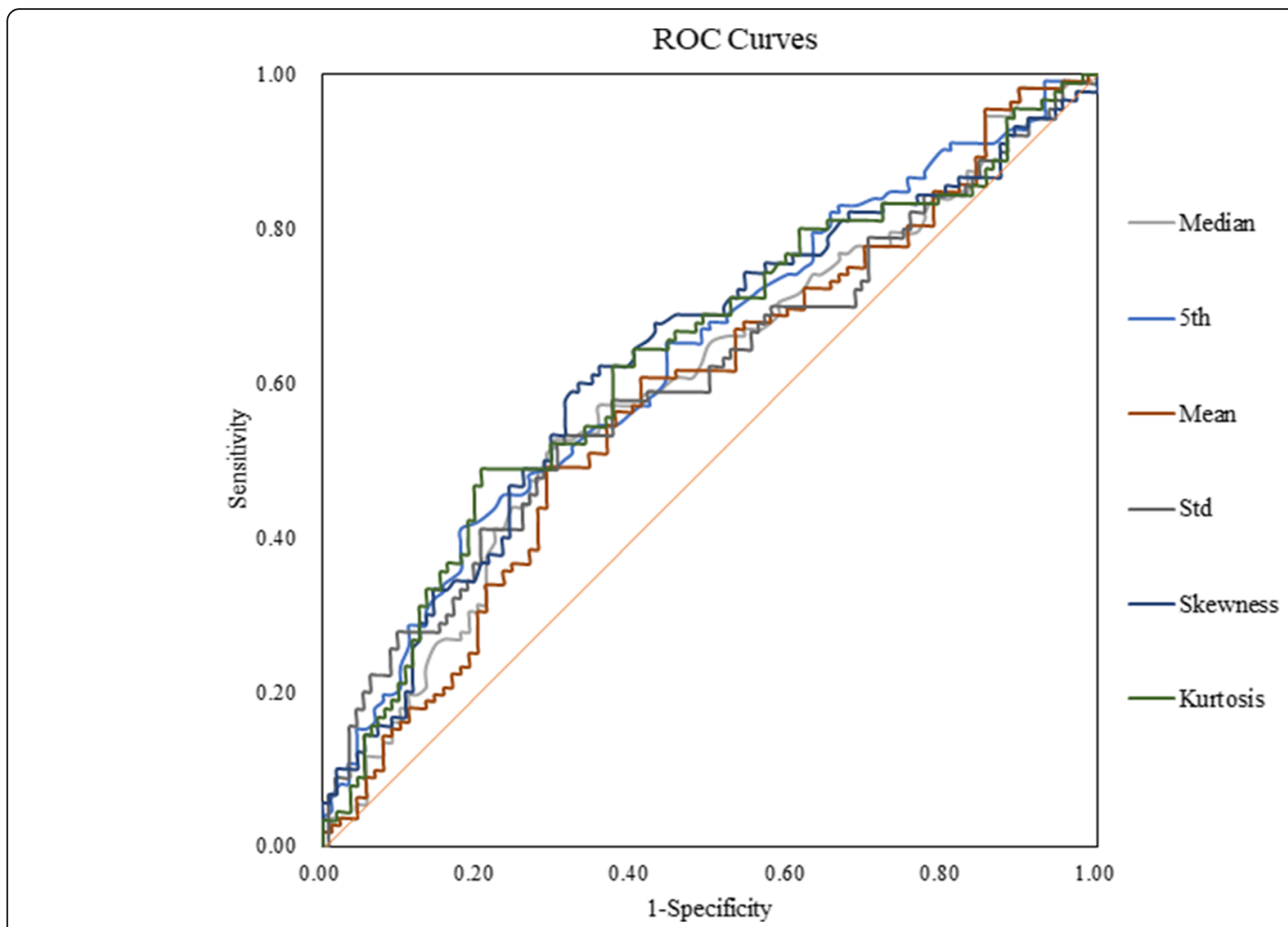

Fig. 5 Receiver operating characteristic (ROC) curves show the diagnostic performance in being able to distinguish prostatic carcinoma from benign prostatic with different T2W image histogram parameters

32]. Absolute $\mathrm{ADC}$ values can vary depending on the choice and number of $b$ values selected. Thus, the current guidelines do not recommend using a single quantitative ADC parameter to characterize lesions [33]. ADC values have also shown reasonable repeatability in vivo, with a variation of about $20 \%$. In these studies, evaluations of mean or median ADCs were the primary focus [34, 35]. A few studies have demonstrated the mean relative percentage variations in ADC of prostate ranging from 6.45 to $15.93 \%$ during single scanning session or when two scans were performed within 2 weeks $[36,37]$.

ADC map histogram and texture parameters showed good diagnostic capabilities in detecting and characterizing diseases and evaluating therapeutic responses. These parameters can determine the spatial variations of ADC values and provide additional information about tumor heterogeneity, which could better reflect tumor characteristics than simply averaging these differences with ROIs. In this study, we found that texture features extracted from ADC maps of prostatic MRI could be used as potential biomarkers to distinguish $\mathrm{BPH}$ from $\mathrm{PCa}$ tumors. Although this is a preliminary study, the imaging phenotype based on the whole-lesion histograms of MR multi-parametric maps might provide as a noninvasive tool to evaluate the biological characteristics and heterogeneity of PCa.

T2WI has been used to show prostatic zonation anatomies to localize lesions, which has served as the key protocol for prostatic MRI since it was first described in the early $1980 \mathrm{~s}$ [38]. Tan et al. performed a metaanalysis and reported that the overall sensitivity and specificity of PCa detection using T2WI were $0.57-0.62$ and

Table 4 Receiver operating characteristic curve results regarding the qualitative analysis of the combined parameters to distinguish prostate cancer from benign prostatic hyperplasia

\begin{tabular}{|c|c|c|c|c|c|c|c|c|}
\hline Parameters & AUC $(95 \% \mathrm{Cl})$ & Sensitivity (\%) & Specificity (\%) & Cutoff value & $p$ value & Youden index & $+\mathrm{LR}$ & $\overline{-L R}$ \\
\hline $\mathrm{ADC}_{5 \text { th }} \& \mathrm{~T} 2 \mathrm{~W}_{\text {Kurtosis }}$ & $0.906(0.857,0.943)$ & 83.3 & 89.3 & $>0.53384$ & $<0.0001$ & 0.7262 & 7.78 & 0.19 \\
\hline$A D C_{5 \text { th }} \& A D C_{\text {Diff-Variance }}$ & $0.906(0.857,0.942)$ & 83.3 & 89.3 & $>0.53566$ & $<0.0001$ & 0.7262 & 7.78 & 0.19 \\
\hline$T 2 W_{5 \text { th }} \& T 2 W_{\text {Diff - Variance }}$ & $0.633(0.562,0.699)$ & 83.3 & 42.9 & $>0.38111$ & 0.0007 & 0.2619 & 1.46 & 0.39 \\
\hline
\end{tabular}

$A D C$ apparent diffusion coefficient, $T 2 W$ T2-weighted imaging, 5 th $5^{\text {th }}$ percentile, Diff-variance difference in variance 


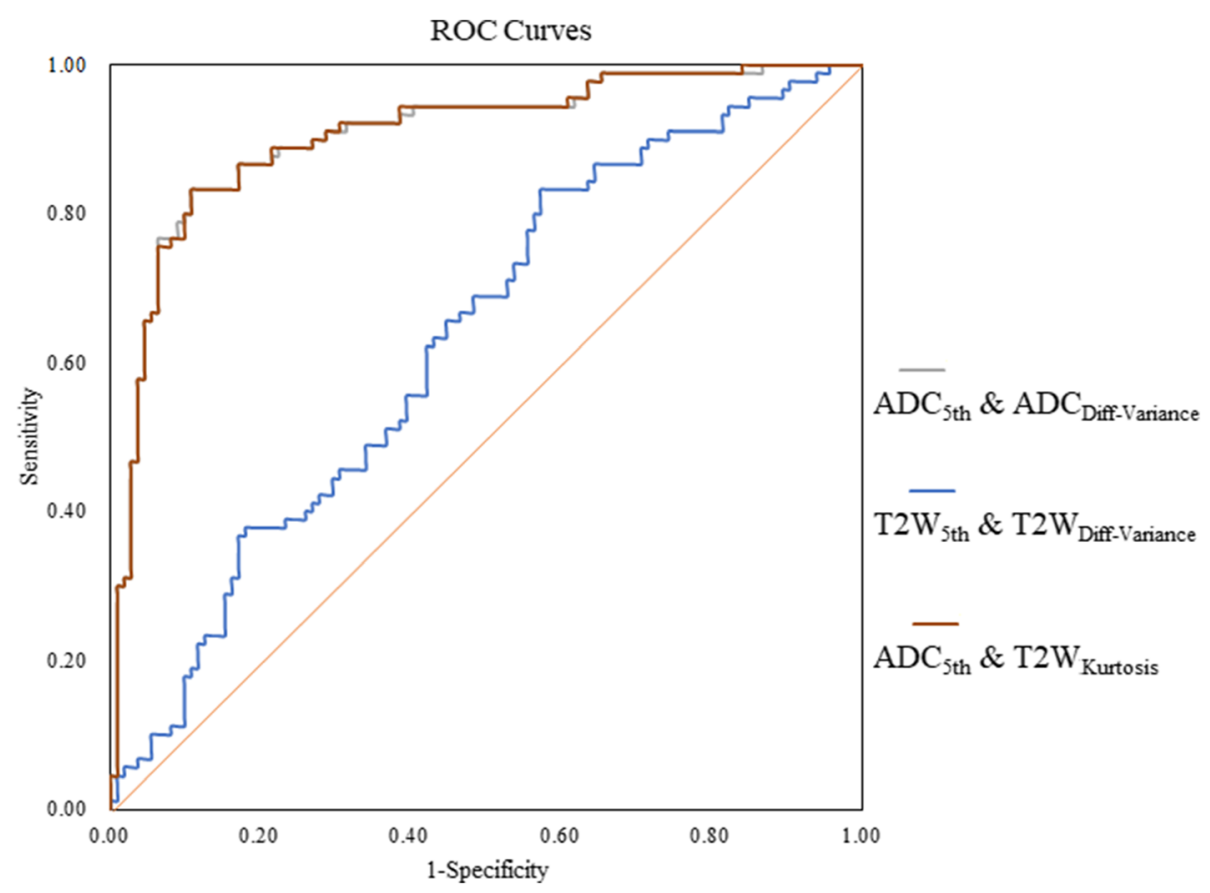

Fig. 6 Receiver operating characteristic (ROC) curves show the diagnostic performance in being able to distinguish prostatic carcinoma from benign prostatic with different combined ADC map histogram parameters. This graph shows improved sensitivity and specificity when the $5^{\text {th }}$ percentile of the $A D C$ values $\left(A D C_{5 \text { th }}\right)$ and $A D C$ differential variance $\left(A D C_{\text {Diff-Variance }}\right.$ ) are used together, but this combination is not better than $5^{\text {th }}$ percentile of the $A D C$ maps $\left(A D C_{5 \text { th }}\right)$ parameter used alone (Fig. 4)

0.74-0.78, respectively [39]. Due to its low diagnostic efficacy, T2WI should not be used alone in clinical practice. In thePI-RADS version 2, T2WI is involved in prostatic lesion scoring, but overall lesions are judged primarily based on DWI [40]. This finding is consistent with the differing abilities of T2WI and DWI texture analyses in being able to detect $\mathrm{PCa}$ and $\mathrm{BPH}$ in our study. Downes et al. created a unique histologic subpattern using the standard Gleason grading system, where T2WI was used to evaluate potential histopathologic differences between interstitial and epithelial tumor components [41]. Nketiah et al. showed that T2W image texture features were more sensitive than signal intensities in revealing tissue morphologies and were closely related to potential pathophysiologic changes in $\mathrm{PCa}$ tumors, further improving the existing PCa classification methods [42]. Daniel et al. showed that bpMRI texture analysis could distinguish normal tissues from tumor tissues in patients with androgen deprivation therapy better compared with traditional histogram parameters [43]. Our study revealed that whole-lesion histogram and texture analysis parameters of T2W images could be used to distinguish $\mathrm{PCa}$ from $\mathrm{BPH}$; however, the diagnostic performance was low compared with those of ADC maps.

Texture analysis has been used to diagnose, differentiate, and assesses the types and therapeutic effects of various tumors, including PCa. Studies have shown that histogram and texture analyses of ADCs contributed to the characterization of prostate tumors. Compared with the mean and median ADC values and $90^{\text {th }}$ percentile Kapp values of diffusion kurtosis imaging, the $10^{\text {th }}$ percentile ADC values correlated better with Gleason classifications, and was superior to other DWI parameters in being able to distinguish low-grade and high-grade tumors [31, 44].

Limited whole-lesion histogram analysis has been used to evaluate PCa therapeutic responses [45]. Kyriazi et al. proved that the $25^{\text {th }}$ percentiles of ADC were the best predictor of chemotherapeutic responses in patients with metastatic ovarian cancer and primary peritoneal cancers [46]. Xie et al. used the histogram and texture analyses of ADCs to differentiate triple-negative breast cancers from other subtypes [14]. Another group used DCE MRI and texture analysis to differentiate malignant glioma from glioblastoma [17]. Texture analysis parameters, extracted from T2W images of rectal cancer patients, were also found to be useful imaging biomarkers to assess tumor responses to neoadjuvant chemotherapies [16]. Meng et al. revealed possible T2W and ADC texture parameters that could be used as noninvasive imaging biomarkers for the early detection of recurrence in patients with advanced cervical cancer after radiotherapy. These parameters could help clinicians adjust 
therapeutic strategies and offer more personalized anticancer therapies [15].

For patients with increased PSA levels or clinical indications of disease on digital rectal examinations, transrectal ultrasound-guided biopsies are currently the most accepted definitive diagnostic method; however, biopsy results have been markedly inconsistent with the histopathology of complete prostatic resections. In this study, we used whole-lesion histogram and texture analysis to overcome this issue.

There were several limitations in this study. First, the sample size was relatively small and the diagnostic performance was not validated on an independent dataset in the current study. We will enlarge the sample size and validate it in the future study. Second, this study mainly focused on patients who had histopathological proved to be positive as well as had definitive lesions in MRI, which may introduce a selection bias for these patients. Third, the histopathologic results of most patients were obtained by transrectal ultrasound-guided biopsy; and therefore, a mismatch between the pathologic locations and delineated ROIs was present. In future studies, 3D MRI-guided biopsies might help to obtain accurate matching between MRIs and pathologic locations and improve the repeatability of prostate MR image interpretations using texture analysis results. Fourth, ROIs of the lesions were acquired on DWI images and transferred to ADC maps and T2W images. Finally, it was retrospective study and all cases came from a single center. Future studies will be prospective and have larger sample sizes. Multiple centers and MR vendors will also be assessed to confirm the current findings.

\section{Conclusions}

In conclusion, parameters derived from whole-lesion histogram and texture analyses of ADC maps and T2W images could be used as imaging biomarkers to assess the biologic characteristics of $\mathrm{PCa}$ and $\mathrm{BPH}$ lesions, however, histogram parameters outperformed texture parameters in the diagnostic performance, which could help clinicians differentiate benign and malignant prostate nodules, providing efficient and accurate clinical decisions.

\section{Abbreviations \\ ADC: Apparent diffusion coefficient; AUC: Area under the curve; $\mathrm{BPH}$ : Benign prostatic hyperplasia; DWl: Diffusion-weighted imaging; FOV: Field-of-view; MRI: Magnetic resonance imaging; PCa: Prostate cancer; PSA: Prostate- specific antigen; ROC: Receiver operating characteristic; TR/TE: Repetition time/echo time}

\section{Acknowledgements}

N/A.

\section{Authors' contributions}

$J$, LC and PX conceived of the present idea. LC and PX designed the study. Data acquisition was performed by TW and TS. LC and CM performed the statistical analysis. LC, PX, QY and WP contributed to the data analysis and interpretation. RG and CF provided the software for data analysis. LC and PX were major contributors and contributed equally to writing the manuscript. All the authors read and approved the final manuscript.

\section{Funding}

This work was supported by the National Key Clinical Specialty Discipline Construction Program of China (Grant Number N/A) and the Natural Science Foundation of Shanghai (Grant Number 20ZR1456300).

\section{Availability of data and materials}

The datasets used and/or analyzed during the current study are available from the corresponding author on reasonable request.

\section{Declarations}

Ethics approval and consent to participate

This study was approved by our institutional ethics committee.

\section{Consent for publication}

Written informed consent was obtained from each patient.

\section{Competing interests}

The authors declare that they have no conflict of interest.

\section{Author details}

'Department of Radiology, Changhai Hospital of Shanghai, The Second Military Medical University, No.168 Changhai Road, 200433 Shanghai, China. ${ }^{2}$ Application Predevelopment, Siemens Healthcare, Erlangen, Germany. ${ }^{3} \mathrm{MR}$ Application Development, Siemens Shenzhen Magnetic Resonance Ltd, Shenzhen, China.

Received: 9 December 2020 Accepted: 3 September 2021

Published online: 27 September 2021

\section{References}

1. Torre LA, Siegel RL, Ward EM, Jemal A. Global Cancer Incidence and mortality rates and trends-an update. Cancer Epidemiol Biomarkers Prev. 2016;25:16-27.

2. Ahmed HU, El-Shater Bosaily A, Brown LC, Gabe R, Kaplan R, Parmar MK, Collaco-Moraes Y, Ward K, Hindley RG, Freeman A, et al. Diagnostic accuracy of multi-parametric MRI and TRUS biopsy in prostate cancer (PROMIS): a paired validating confirmatory study. Lancet. 2017;389:815-22.

3. Egger SJ, Calopedos RJ, O'Connell DL, Chambers SK, Woo HH, Smith DP. Long-term psychological and quality-of-life effects of active surveillance and watchful waiting after diagnosis of low-risk localised prostate cancer. Eur Urol. 2018;73:859-67.

4. Wilt TJ, Jones KM, Barry MJ, Andriole GL, Culkin D, Wheeler T, Aronson WJ, Brawer MK. Follow-up of prostatectomy versus observation for early prostate cancer. N Engl J Med. 2017;377:132-42.

5. Miller KD, Siegel RL, Lin CC, Mariotto AB, Kramer JL, Rowland JH, Stein KD, Alteri R, Jemal A. Cancer treatment and survivorship statistics, 2016. CA Cancer J Clin. 2016;66:271-89.

6. Tamada T, Kido A, Takeuchi M, Yamamoto A, Miyaji Y, Kanomata N, Sone T. Comparison of PI-RADS version 2 and PI-RADS version 2.1 for the detection of transition zone prostate cancer. Eur J Radiol. 2019;121:108704.

7. Mazzoni LN, Lucarini S, Chiti S, Busoni S, Gori C, Menchi I. Diffusionweighted signal models in healthy and cancerous peripheral prostate tissues: comparison of outcomes obtained at different b-values. J Magn Reson Imaging. 2014;39:512-8.

8. Surov A, Meyer HJ, Wienke A. Correlations between apparent diffusion coefficient and gleason score in prostate cancer: a systematic Review. Eur Urol Oncol. 2019. https://doi.org/10.1016/j.euo.2018.12.006.

9. Kuhl CK, Bruhn R, Kramer N, Nebelung S, Heidenreich A, Schrading S. Abbreviated biparametric prostate MR imaging in men with elevated prostate-specific antigen. Radiology. 2017;285:493-505.

10. Vargas HA, Hotker AM, Goldman DA, Moskowitz CS, Gondo T, Matsumoto K, Ehdaie B, Woo S, Fine SW, Reuter VE, et al. Updated prostate imaging reporting and data system (PIRADS V2) recommendations for the detection of clinically significant prostate cancer using multiparametric MRI: critical 
evaluation using whole-mount pathology as standard of reference. Eur Radiol. 2016;26:1606-12.

11. Rosenkrantz $A B$, Ginocchio LA, Cornfeld D, Froemming AT, Gupta RT, Turkbey B, Westphalen AC, Babb JS, Margolis DJ. Interobserver reproducibility of the PI-RADS Version 2 Lexicon: a multicenter study of six experienced prostate radiologists. Radiology. 2016;280:793-804.

12. Ganeshan B, Miles KA. Quantifying tumour heterogeneity with $C T$. Cancer Imaging. 2013;13:140-9.

13. Davnall F, Yip CS, Ljungqvist G, Selmi M, Ng F, Sanghera B, Ganeshan B, Miles KA, Cook GJ, Goh V. Assessment of tumor heterogeneity: an emerging imaging tool for clinical practice? Insights Imaging. 2012;3:573-89.

14. Xie T, Zhao Q, Fu C, Bai Q, Zhou X, Li L, Grimm R, Liu L, Gu Y, Peng W. Differentiation of triple-negative breast cancer from other subtypes through whole-tumor histogram analysis on multiparametric MR imaging. Eur Radiol. 2019:29:2535-44.

15. Meng J, Liu S, Zhu L, Zhu L, Wang H, Xie L, Guan Y, He J, Yang X, Zhou Z. Texture analysis as imaging biomarker for recurrence in advanced cervical cancer treated with CCRT. Sci Rep. 2018;8:11399.

16. De Cecco CN, Ganeshan B, Ciolina M, Rengo M, Meinel FG, Musio D, De Felice F, Raffetto N, Tombolini V, Laghi A. Texture analysis as imaging biomarker of tumoral response to neoadjuvant chemoradiotherapy in rectal cancer patients studied with 3-T magnetic resonance. Invest Radiol. 2015;50: 239-45.

17. Eliat PA, Olivie D, Saikali S, Carsin B, Saint-Jalmes H, de Certaines JD. Can dynamic contrast-enhanced magnetic resonance imaging combined with texture analysis differentiate malignant glioneuronal tumors from other glioblastoma? Neurol Res Int. 2012;2012:195176.

18. Sidhu HS, Benigno S, Ganeshan B, Dikaios N, Johnston EW, Allen C, Kirkham A, Groves AM, Ahmed HU, Emberton M, et al. Textural analysis of multiparametric MRI detects transition zone prostate cancer. Eur Radiol. 2017;27:2348-58

19. Wibmer A, Hricak H, Gondo T, Matsumoto K, Veeraraghavan $H$, Fehr $D$, Zheng J, Goldman D, Moskowitz C, Fine SW, et al. Haralick texture analysis of prostate MRl: utility for differentiating non-cancerous prostate from prostate cancer and differentiating prostate cancers with different Gleason scores. Fur Radiol. 2015:25:2840-50

20. Sun K, Zhu H, Chai W, Zhan Y, Nickel D, Grimm R, Fu C, Yan F. Whole-lesion histogram and texture analyses of breast lesions on inline quantitative DCE mapping with CAIPIRINHA-Dixon-TWIST-VIBE. Eur Radiol. 2020;30:57-65.

21. Enkhbaatar NE, Inoue S, Yamamuro H, Kawada S, Miyaoka M, Nakamura N, Sadahiro S, Imai Y. MR Imaging with apparent diffusion coefficient histogram analysis: evaluation of locally advanced rectal cancer after chemotherapy and radiation therapy. Radiology. 2018;288:129-37.

22. Cui Y, Li C, Liu Y, Jiang Y, Yu L, Liu M, Zhang W, Shi K, Zhang C, Zhang J, et al. Differentiation of prostate cancer and benign prostatic hyperplasia: comparisons of the histogram analysis of intravoxel incoherent motion and monoexponential model with in-bore MR-guided biopsy as pathological reference. Abdom Radiol (NY). 2020;45:3265-77.

23. Chatterjee A, Gallan AJ, He D, Fan X, Mustafi D, Yousuf A, Antic T, Karczmar GS, Oto A. Revisiting quantitative multi-parametric MRI of benign prostatic hyperplasia and its differentiation from transition zone cancer. Abdom Radiol (NY). 2019;44:2233-43.

24. Bonekamp D, Kohl S, Wiesenfarth M, Schelb P, Radtke JP, Gotz M, Kickingereder P, Yaqubi K, Hitthaler B, Gahlert N, et al. Radiomic machine learning for characterization of prostate lesions with MRI: comparison to ADC values. Radiology. 2018;289:128-37.

25. Peng $Y$, Jiang $Y$, Yang C, Brown JB, Antic T, Sethi I, Schmid-Tannwald C, Giger ML, Eggener SE, Oto A. Quantitative analysis of multiparametric prostate MR images: differentiation between prostate cancer and normal tissue and correlation with Gleason score-a computer-aided diagnosis development study. Radiology. 2013;267:787-96.

26. Egevad L, Delahunt B, Srigley JR, Samaratunga H. International Society of Urological Pathology (ISUP) grading of prostate cancer - An ISUP consensus on contemporary grading. APMIS. 2016;124:433-5.

27. Hanley JA, McNeil BJ. The meaning and use of the area under a receiver operating characteristic (ROC) curve. Radiology. 1982;143:29-36.

28. Zweig MH, Campbell G. Receiver-operating characteristic (ROC) plots: a fundamental evaluation tool in clinical medicine. Clin Chem. 1993;39:56177.

29. Koh DM, Collins DJ. Diffusion-weighted MRI in the body: applications and challenges in oncology. AJR Am J Roentgenol. 2007;188:1622-35.
30. Barrett T, Priest AN, Lawrence EM, Goldman DA, Warren AY, Gnanapragasam VJ, Sala E, Gallagher FA. Ratio of tumor to normal prostate tissue apparent diffusion coefficient as a method for quantifying DWI of the prostate. AJR Am J Roentgenol. 2015;205:W585-93.

31. Donati OF, Mazaheri Y, Afaq A, Vargas HA, Zheng J, Moskowitz CS, Hricak H, Akin O. Prostate cancer aggressiveness: assessment with whole-lesion histogram analysis of the apparent diffusion coefficient. Radiology. 2014;271: $143-52$.

32. Nagarajan R, Margolis D, Raman S, Sheng K, King C, Reiter R, Thomas MA. Correlation of Gleason scores with diffusion-weighted imaging findings of prostate cancer. Adv Urol. 2012;2012:374805.

33. Brizmohun Appayya M, Adshead J, Ahmed HU, Allen C, Bainbridge A, Barrett T, Giganti F, Graham J, Haslam P, Johnston EW, et al. National implementation of multi-parametric magnetic resonance imaging for prostate cancer detection - recommendations from a UK consensus meeting. BJU Int. 2018;122:13-25.

34. Rosenkrantz AB, Oei M, Babb JS, Niver BE, Taouli B. Diffusion-weighted imaging of the abdomen at 3.0 Tesla: image quality and apparent diffusion coefficient reproducibility compared with 1.5 Tesla. J Magn Reson Imaging. 2011;33:128-35.

35. Miquel ME, Scott AD, Macdougall ND, Boubertakh R, Bharwani N, Rockall AG. In vitro and in vivo repeatability of abdominal diffusion-weighted MRI. Br J Radiol. 2012;85:1507-12.

36. Sadinski M, Medved M, Karademir I, Wang S, Peng Y, Jiang Y, Sammet S, Karczmar G, Oto A. Short-term reproducibility of apparent diffusion coefficient estimated from diffusion-weighted MRI of the prostate. Abdom Imaging. 2015;40:2523-8.

37. Fedorov A, Vangel MG, Tempany CM, Fennessy FM. Multiparametric magnetic resonance imaging of the prostate: repeatability of volume and apparent diffusion coefficient quantification. Invest Radiol. 2017;52:538-46.

38. Steyn JH, Smith FW. Nuclear magnetic resonance (NMR) imaging of the prostate. Br J Urol. 1984;56:679-81.

39. Tan $\mathrm{CH}$, Wei W, Johnson V, Kundra V. Diffusion-weighted MRI in the detection of prostate cancer: meta-analysis. AJR Am J Roentgenol. 2012;199: 822-9.

40. Weinreb JC, Barentsz JO, Choyke PL, Cornud F, Haider MA, Macura K, Margolis D, Schnall MD, Shtern F, Tempany CM, et al. PI-RADS prostate imaging - reporting and data system: 2015, Version 2. Eur Urol. 2016;69:1640.

41. Downes MR, Gibson E, Sykes J, Haider M, van der Kwast TH, Ward A. Determination of the association between T2-weighted MRI and Gleason sub-pattern: a proof of principle study. Acad Radiol. 2016;23:1412-21.

42. Nketiah G, Elschot M, Kim E, Teruel JR, Scheenen TW, Bathen TF, Selnaes KM. T2-weighted MRI-derived textural features reflect prostate cancer aggressiveness: preliminary results. Eur Radiol. 2017;27:3050-9.

43. Daniel M, Kuess P, Andrzejewski P, Nyholm T, Helbich T, Polanec S, Dragschitz F, Goldner G, Georg D, Baltzer P. Impact of androgen deprivation therapy on apparent diffusion coefficient and T2w MRI for histogram and texture analysis with respect to focal radiotherapy of prostate cancer. Strahlenther Onkol. 2019;195:402-11.

44. Wang Q, Li H, Yan X, Wu CJ, Liu XS, Shi HB, Zhang YD. Histogram analysis of diffusion kurtosis magnetic resonance imaging in differentiation of pathologic Gleason grade of prostate cancer. Urol Oncol. 2015;33:337 e1524.

45. Rosenkrantz AB, Ream JM, Nolan P, Rusinek H, Deng FM, Taneja SS. Prostate cancer: utility of whole-lesion apparent diffusion coefficient metrics for prediction of biochemical recurrence after radical prostatectomy. AJR Am J Roentgenol. 2015;205:1208-14.

46. Kyriazi S, Collins DJ, Messiou C, Pennert K, Davidson RL, Giles SL, Kaye SB, Desouza NM. Metastatic ovarian and primary peritoneal cancer: assessing chemotherapy response with diffusion-weighted MR imaging-value of histogram analysis of apparent diffusion coefficients. Radiology. 2011;261: 182-92.

\section{Publisher's Note}

Springer Nature remains neutral with regard to jurisdictional claims in published maps and institutional affiliations. 